\title{
Time-Resolved Spectroscopy with SDSS
}

\author{
Steven Bickerton ${ }^{1}$, Carles Badenes ${ }^{2}$, Thomas Hettinger ${ }^{3}$, Timothy \\ Beers $^{3}$, and Sonya Huang ${ }^{1}$ \\ ${ }^{1}$ Dept. of Astrophysical Sciences, Princeton University, Princeton, NJ 08544, USA \\ email: bick@astro.princeton.edu \\ ${ }^{2}$ Dept of Physics and Astronomy, University of Pittsburgh, Pittsburgh, PA 15260, USA \\ email: badenes@pitt.edu \\ ${ }^{3}$ Dept of Physics and Astronomy and JINA: Joint Institute for Nuclear Astrophysics, \\ Michigan State University, East Lansing, MI 48824, USA \\ email: hettin12@msu.edu, beers@pa.msu.edu
}

\begin{abstract}
We present a brief technical outline of the newly-formed project, "Detection of Spectroscopic Differences over Time" (DS/DT). Our collaboration is using individual exposures from the SDSS spectroscopic archive to produce a uniformly-processed set of time-resolved spectra. Here we provide an overview of the properties and processing of the available data, and highlight the wide range of time base-lines present in the archive.
\end{abstract}

Keywords. techniques: spectroscopic, methods:data analysis, surveys, stars: variable: other

\section{Introduction}

The Sloan Digital Sky Survey (SDSS; York et al. 2000) has been in operation for over a decade, and through three separate phases (SDSS I, II, and III) has accumulated an extensive archive of photometric and spectroscopic astrophysical observations. Here we describe a newly-formed data mining collaboration, the "Detection of Spectroscopic Differences over Time" or DS/DT project, to search for variability in SDSS spectra.

Though cadences and exposure times varied, the SDSS spectra were generally observed on three or more occasions, with exposure times of at least 900s. These individual exposures were combined to produce the final spectra released to the community.

Only a handful of groups have examined the sub-spectra for evidence of variability: Hilton et al. (2010) examined flaring in M-dwarfs, while Mullally et al. (2009) and Badenes et al. (2009) used radial-velocity shifts to identify WD-WD binaries. Radial velocities have also been used by Rebassa-Mansergas et al. (2010) to identify binaries. With such an enormous diversity of objects, from QSOs and AGNs to stars of all spectral types, there is a vast amount of time-variable data which have never been evaluated.

The primary objective of our work is to produce a uniformly-processed archive of the individual sub-spectra for all SDSS spectroscopic observations. We are developing a datamining pipeline to target some specific forms of variability (radial velocities, flaring, etc) as well as serendipitous anomalous variability.

\section{Data Processing}

Our pipeline is an extension of the SDSS spectroscopic pipeline, spectro, used to produce the seventh data release of the Sloan Digital Sky Survey (Abazajian et al. 2009). The original spectro pipeline consists of three principle stages for (1) extraction and calibration of the raw spectroscopic traces, (2) stacking the sub-spectra and stitching 
the data from the red and blue cameras, and (3) object classification and redshift determination. We have written a modified 2nd stage to perform only the red/blue stitching for the individual exposures. Our new combined step generates individual (per-object) output files containing the full co-added spectra and their associated sub-spectra.

\section{Time-Baselines}

During the SDSS observing programme, a wide range of time base-lines was sampled. A given plate (640 fibers) was typically observed in three back-to-back 900s exposures, and in most cases the base-line is therefore $\lesssim 30$ minutes. However, plates which were incomplete at the end of an observing session were continued the following night, thus giving many plates a base-line of $>12$ hours. In some cases, previously-observed plates were later replugged for further observation, generating base-lines of several weeks or sometimes much longer. Finally, some targets were observed on multiple plates, again providing base-lines from weeks to months. Figure 1 shows histograms of the time baselines sampled (measured between the mid-points of the first and last available exposures) and the number of exposures. The sample includes the SDSS I and II spectra, and the SEGUE-II subset of the SDSS III spectra.
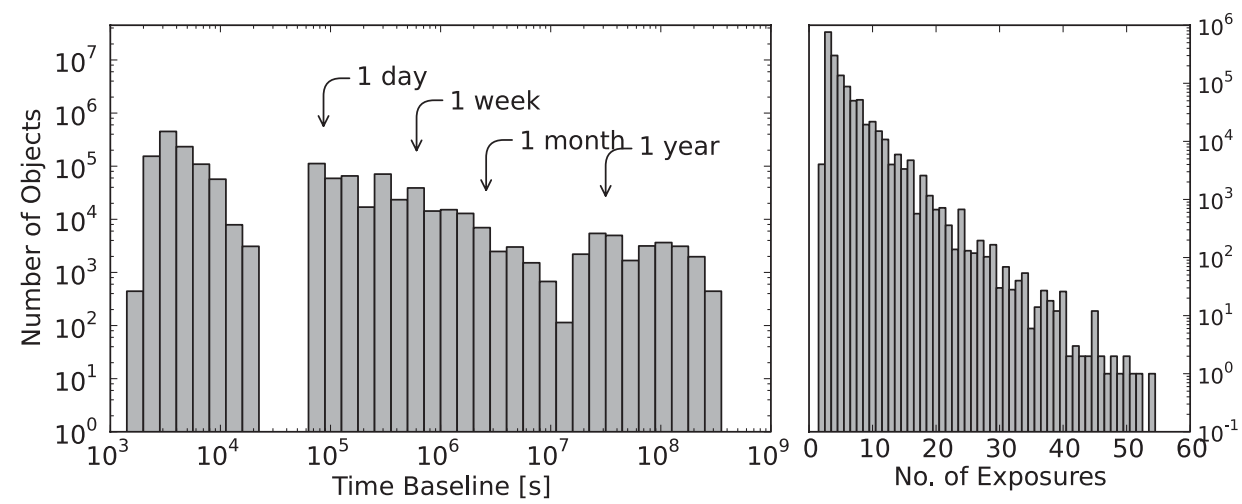

Figure 1. The per-object time base-lines sampled by the SDSS sub-spectra (left), and the number of targets having a given number of exposures available (right).

\section{Summary}

The DS/DT project is a newly-formed data-mining project to explore time variability in the sub-spectra of the SDSS spectroscopic archive. We have developed a branch pipeline to process and collate the sub-spectra for each target into individual FITS files. It is our intention to make our full data set publicly available. Those interested in testing and providing feedback on our preliminary data are invited to contact us.

\section{References}

Abazajian, K. N., et al. 2009, ApJS, 182, 543

Badenes, C., Mullally, F., Thompson, S. E., \& Lupton, R. H. 2009, ApJ, 707, 971

Hilton, E. J., West, A. A., Hawley, S. L., \& Kowalski, A. F. 2010, AJ, 140, 1402

Mullally, F., Badenes, C., Thompson, S. E., \& Lupton, R. 2009, ApJL, 707, L51

Rebassa-Mansergas, A., et al. 2010, MNRAS, 402, 620

York, D. G., et al. 2000, AJ, 120, 1579 